\title{
A RARE PRESENTATION OF SCRUB TYPHUS AS ADEM
}

\author{
D. Rajesekar1, V. Abitha², Noorul Ameen ${ }^{3}$
}

1 Professor and HOD, Department of General Medicine, Chettinad Health and Research Institute, Chennai. 2Postgraduate Student, Department of General Medicine, Chettinad Health and Research Institute, Chennai. ${ }^{3}$ Assistant Professor, Department of General Medicine, Chettinad Health and Research Institute, Chennai.

\section{ABSTRACT}

\section{BACKGROUND}

A young male presented with fever, chills, neck rigidity, unsteadiness and abdominal pain. Patient had signs of chorea (hung-up reflex, exaggerated abdominal reflex), signs of meningoencephalitis (Kernig's positive, opsoclonus) generalised lymphadenopathy (involving cervical, inguinal \& axillary regions)-tender \& matted, eschar in right inguinal region, left UMN type of facial nerve palsy, signs of pyramidal tract (exaggerated deep tendon reflexes, right plantar extensor), signs of extrapyramidal tract (cog-wheel rigidity) and signs of cerebellar signs (hypotonia \& stance and gait ataxia) were present bilaterally giving a picture of Acute Disseminated Encephalomyelitis (ADEM). ELISA for scrub typhus IgM was positive after ruling out malaria, dengue, leptospirosis, bacterial, viral and TB meningitis. ADEM is most rare and deadly presentation of Scrub typhus.

\section{KEYWORDS}

Scrub Typhus, Trombiculid Mite, Opsoclonus, ADEM.

HOW TO CITE THIS ARTICLE: Rajesekar D, Abitha V, Ameen N. A rare presentation of scrub typhus as ADEM. J. Evolution Med. Dent. Sci. 2017;6(26): 2211-2212, DOI: 10.14260/Jemds/2017/477

\section{BACKGROUND}

Scrub typhus is an acute febrile illness caused by Orientia tsutsugamushi, an obligate intracellular Gram-negative bacterium and spread by transovarial transmission in trombiculid mites. They usually feed on rats, their reservoir hosts. These happen to infest humans by accident, when they come in contact with mite-infested scrub vegetation during the wet season. ${ }^{1}$ Scrub typhus is also commonly reported from various parts of southern India. ${ }^{1}$ The symptoms commonly seen are fever, headache, myalgia, nausea and vomiting, rash, jaundice, adenopathy, etc. Identification of the eschar, at the site of the larval feed has the most important diagnostic role in the disease and is not easy to find in dark skinned people. The primary site of attack are the vascular endothelial cells ${ }^{2}$ leading to pathogenesis of multiple organ damage. Complications usually develop after the first week of the disease, like acute renal failure, hepatitis, disseminated intravascular, adult respiratory distress syndrome, multiorgan dysfunction, and so on. Many neurological manifestations like meningitis, ${ }^{2}$ encephalomyelitis, ${ }^{3}$ demyelination, ${ }^{4}$ meningoencephalitis ${ }^{5}$, etc, have also been reported. Here we have a rare presentation of Scrub typhus as ADEM (Acute Disseminated Encephalomyelitis).

\section{Case Study}

Patient came with complaints of high grade fever with chills, vomiting since 1 week and abdominal pain and generalised weakness and unsteadiness. Patient was crying and restless. On examination, patient was conscious, disturbed, afebrile, pulse rate-73, BP- 110/80, capillary blood glucose- $80 \mathrm{mg} / \mathrm{dL}$,

Financial or Other, Competing Interest: None.

Submission 13-02-2017, Peer Review 09-03-2017,

Acceptance 25-03-2017, Published 30-03-2017.

Corresponding Author:

Dr. D. Rajesekar,

Kelambakkam, OMR,

Chettinad Health and Research Institute,

Chennai.

E-mail: okazhaki87@gmail.com

DOI: $10.14260 /$ jemds $/ 2017 / 477$

\section{(c) $(1) \odot$}

$\mathrm{SPO}_{2}-97 \%$, episcleritis seen, opsoclonus present, generalised lymphadenopathy (cervical/inguinal/axillary)-tender and matted, eschar in right inguinal region seen, central nervous system - emotional liability present, left upper motor neuron type of facial nerve palsy, exaggerated deep tendon reflexes, exaggerated abdominal reflex, right plantar extensor, cogwheel rigidity and stance and gait ataxia were present. Bilateral cerebellar signs dysmetria and heel-shin test and dysdiadochokinesia were positive, bilateral hypotonia present in upper and lower limbs, sensory system was intact, bowel and bladder- not involved. Investigations showed haemoglobin-15.8, PCV-49, Leucocytosis $(20,100)$, neutropenia (18.3), lymphocytosis $(70 \%)$, monocytosis (11\%), platelets-1.94 cells/cu.mm, renal parameters and electrolytes were normal; liver enzymes elevated (AST-89, ALT- 74, LDH - 598). Chest X-ray showed increased bronchovascular markings. Urine culture \& blood culture - no growth, HIV - nonreactive, HbsAg - negative, HCVNonreactive, serum procalcitonin - positive $(>0.54 \mathrm{ng} / \mathrm{mL})$, dengue IgM \& IgG \& NS1 antigen-negative, Lepto IgM negative. Smear MP \& MF - negative. Scrub typhus (ELISA) IgM -Reactive. CSF study: proteins- $146.3 \mathrm{mg} / \mathrm{dL}$, glucose- 45 $\mathrm{mg} / \mathrm{dL}$, chloride- $114 \mathrm{mEq} / \mathrm{L}$, TC- 50 cells. Patient was started with doxycycline $100 \mathrm{mg}$ twice daily and all manifestations disappeared gradually.

\section{DISCUSSION}

Scrub typhus is most common in wet season (October to February $)^{5}$ in India and the eschar will usually be seen in moist regions like groin, axilla, loin, etc. Meningoencephalitis with altered liver enzymes should raise suspicion for Scrub typhus especially after ruling out TB Meningitis. Presence of eschar may give a clue to diagnosis, common sites of eschar in female are chest (inframammary region) and abdomen and common sites in male are axilla, groin and genitalia; other unusual sites are cheek, ear lobe and dorsum of feet. 6 But it is present in only 20-30 [percent] and usually goes unnoticed in dark skinned people. It can mimic TB meningitis and starting anti-tubercular treatment can worsen liver functions. Laboratory tests and other investigations that help diagnosis are Scrub IgM and IgG, Indirect Immunofluorescence test, 
PCR of specific genomic type, MRI brain in some studies showed diffuse cerebral oedema along with $\mathrm{T} 2$ weighted and FLAIR images of hyperintensities in regions of putamen and thalamus.7-8 The triad of features of fever, lymphadenopathy and eschar should raise a suspicion of Scrub typhus especially during the season. ${ }^{9}$ We should look out for other complications like acute kidney injury, 7 septic shock, ${ }^{10}$ respiratory failure 11 and Guillain-Barre Syndrome.11 GBS secondary to scrub typhus is suspected to be due to a phenomenon called molecular mimicry, which is cross reactivity between the host's myelin cells or peripheral nerve axons with 0 . tsutsugamushi antibody or antigens presented on infected cells. Both the host and the pathogen trigger the receptors on $\mathrm{T}$ or $\mathrm{B}$ lymphocyte to activate this reaction. It is confirmed by GD1b and GM1 IgM anti-glucoside antibodies in serum. 11 This might as well apply to any neurological tissue involved.

\section{CONCLUSION}

Early diagnosis and treatment with doxycycline is necessary to prevent life threatening complications (Doxycycline 100 milligrams twice daily for 7 days). Studies also report azithromycin as an efficient alternative to doxycycline ${ }^{12}$ in patients who are unable to tolerate or in pregnant women where it is contraindicated, in children less than 8 years or in doxycycline-resistant Scrub typhus prevalent areas. 12

\section{REFERENCES}

[1] Neyaz Z, Bhattacharya V, Muzaffar N, et al. Brain MRI findings in a patient with scrub typhus infection. Neurology India 2016;64(4):788-92.

[2] Pai H, Sohn S, Seong Y, et al. Central nervous system involvement in patients with scrub typhus. Clinical Infectious Diseases 1997;24(3):436-40.

[3] Kim DE, Lee SH, Park KI, et al. Scrub typhus encephalomyelitis with prominent focal neurologic signs. Archives of Neurology 2000;57(12):1770-2.
[4] Rosenblum MJ, Masland RL, Harrell GT. Residual effects of rickettsial disease on the central nervous system: results of neurologic examinations and electroencephalograms following Rocky Mountain spotted fever. AMA Archives of Internal Medicine 1952;90(4):444-55.

[5] Mathai E, Rolain JM, Verghese GM, et al. Outbreak of scrub typhus in southern India during the cooler months. Annals of the New York Academy of Sciences 2003;990(1):359-64.

[6] Kundavaram AP, Jonathan AJ, Nathaniel SD, et al. Eschar in scrub typhus: a valuable clue to the diagnosis. Journal of Postgraduate Medicine 2013;59(3):177-8.

[7] Pradhan B, Jindal A. Acute encephalitis syndrome following scrub typhus infection. Indian Journal of Critical Care Medicine 2014;18(10):700-1.

[8] Chua CJ, Tan KS, Ramli N, et al. Scrub typhus with central nervous system involvement: a case report with CT and MR imaging features. Neurol J South East Asia 1999;4:53-7.

[9] Ali A, Haridasan RK, Kumar SKK, et al. A study on scrub typhus in a tertiary care hospital. Kerala Medical Journal 2009;2(1):7-9.

[10] Thap LC, Supanaranond W, Treeprasertsuk S, et al. Septic shock secondary to scrub typhus: characteristics and complications. Southeast Asian J Trop Med Public Health 2002;33(4):780-6.

[11] Ju IN, Lee JW, Cho SY, et al. Two cases of scrub typhus presenting with Guillain-Barré syndrome with respiratory failure. The Korean Journal of Internal Medicine 2011;26(4):474-6.

[12] Phimda K, Hoontrakul S, Suttinont C, et al. Doxycycline versus azithromycin for treatment of leptospirosis and scrub typhus. Antimicrobial Agents and Chemotherapy 2007;51(9):3259-63. 